\title{
Automatic Synthesis of Both the Control Law and Parameters for a Controller for a Three-Lag Plant with Five-Second Delay using Genetic Programming and Simulation Techniques
}

\author{
John R. Koza \\ Stanford University \\ Stanford, California \\ kozaestanford.edu
}

\author{
Martin A. Keane \\ Econometrics Inc. \\ Chicago, Illinois \\ makeanedix.netcom.com
}

\author{
Jessen Yu \\ Genetic Programming Inc. \\ Los Altos, California \\ jyuecs.stanford.edu
}

\author{
William Mydlowec \\ Genetic Programming Inc. \\ Los Altos, California \\ mydecs.stanford.edu
}

\begin{abstract}
This paper describes how the process of synthesizing the design of both the topology (control law) and the numerical parameter values (tuning) for a controller can be automated using genetic programming. Genetic programming can be used to automatically make the decisions concerning the total number of signal processing blocks to be employed in a controller, the type of each block, the topological interconnections between the blocks, and the values (tuning) of all parameters for all blocks requiring parameters. In synthesizing the design of controllers, genetic programming can simultaneously optimize prespecified performance metrics (such as minimizing the time required to bring the plant output to the desired value), satisfy time-domain constraints (such as overshoot and disturbance rejection), and satisfy frequency domain constraints. Evolutionary methods have the advantage of not being encumbered by preconceptions that limit its search to well-traveled paths. Genetic programming is applied to an illustrative problem involving the design of a controller for a three-lag plant with a significant (fivesecond) time delay in the external feedback from the plant to the controller. The delay in the feedback makes the design of an effective controller difficult.
\end{abstract}

\section{Introduction}

The process of creating (synthesizing) the design of a controller entails making decisions concerning the total number of signal processing blocks to be employed in the controller, the type of each block (e.g., lead, lag, gain, integrator, differentiator, adder, inverter, subtractor, and multiplier), the values (tuning) of all parameters for all blocks requiring parameters, and the topological interconnections between the blocks. The latter includes the question of whether or not to employ internal feedback (i.e., feedback inside the controller).
Forrest H Bennett III

Genetic Programming Inc. (Currently, FX Palo Alto Laboratory, Palo Alto, California)

bennett@pal.xerox.com

The problem of synthesizing a controller to satisfy prespecified requirements is sometimes solvable by analytic techniques (often oriented toward producing conventional PID controllers). However, as Boyd and Barratt stated in Linear Controller Design: Limits of Performance (1991),

"The challenge for controller design is to productively use the enormous computing power available. Many current methods of computer-aided controller design simply automate procedures developed in the 1930's through the 1950's ..."

This paper describes how genetic programming can be used to automatically create both the topology and the numerical parameter values for a controller directly from a high-level statement of the requirements of the controller. Genetic programming can, if desired, simultaneously optimize prespecified performance metrics (such as minimizing the time required to bring the plant output to the desired value as measured by, say, the integral of the time-weighted absolute error), satisfy timedomain constraints (involving, say, overshoot and disturbance rejection), and satisfy frequency domain constraints. Evolutionary methods have the advantage of not being encumbered by preconceptions that limit their search to well-traveled paths.

Section 2 describes an illustrative problem of controller synthesis. Section 3 provides general background on genetic programming. Section 4 describes how genetic programming is applied to control problems. Section 5 describes the preparatory steps necessary to apply genetic programming to the illustrative control problem. Section 6 presents the results.

\section{$2 \quad$ Illustrative Problem}

The illustrative problem entails creation of both the topology and parameter values for a controller for a threelag plant with a significant (five-second) time delay in the external feedback from the plant output to the controller such that plant output reaches the level of the reference signal in minimal time (as measured by the integral of the time-weighted absolute error), such that the overshoot in response to a step input is less than $2 \%$, and such that the 
controller is robust in the face of disturbance (added into the controller output). The delay in the feedback makes the design of an effective controller difficult (Astrom and Hagglund 1995). The transfer function of the plant is

$$
G(s)=\frac{K e^{-5 s}}{(1+\tau s)^{3}}
$$

A controller presented in Astrom and Hagglund 1995 (page 225) delivers credible performance on this problem for values of $K=1$ and $\tau=1$.

To make the statement of the problem more realistic, we added an additional constraint (satisfied by the controller presented in Astrom and Hagglund 1995) that the input to the plant is limited to the range between -40 and +40 volts. Moreover, the plant in this paper operates over several different combinations of values for $K$ and $\tau$ (whereas the controller designed by Astrom and Hagglund was intended only for $K=1$ and $\tau=1$ ).

\section{$3 \quad$ Background on Genetic Programming}

Genetic programming (Koza 1992; Koza and Rice 1992; Koza 1994a, 1994b) is an automatic technique for generating computer programs to solve, or approximately solve, problems. Genetic programming is an extension of the genetic algorithm (Holland 1975). Genetic programming is described in detail in the recent book Genetic Programming III: Darwinian Invention and Problem Solving (Koza, Bennett, Andre, and Keane 1999; Koza, Bennett, Andre, Keane, and Brave 1999).

Genetic programming is capable of evolving reusable, parametrized, hierarchically-called automatically defined functions (ADFs) so that an overall program consists of a main result-producing branch and one or more reusable and parameterizable automatically defined functions (function-defining branches). In addition, architecturealtering operations enable genetic programming to automatically determine the number of automatically defined functions, the number of arguments that each possesses, and the nature of the hierarchical references, if any, among such automatically defined functions.

Genetic programming often creates novel designs because it is a probabilistic process that is not encumbered by the preconceptions that often channel human thinking down familiar paths. For example, genetic programming is capable of synthesizing the design of both the topology and sizing for a wide variety of analog electrical circuits from a high-level statement of the circuit's desired behavior and characteristics (Koza, Bennett, Andre, and Keane 1999; Koza, Bennett, Andre, Keane, and Brave 1999). Five of the evolved analog circuits in that book infringe on previously issued patents while five others deliver the same functionality as previously patented inventions in a novel way.

There are now over two dozen instances (Koza, Keane, Yu, Bennett, and Mydlowec 1999 2000) where genetic programming has produced human-competitive results. These examples involve quantum computing, the annual Robo Cup competition, computational molecular biology, cellular automata, sorting networks, the automatic synthesis of the topology and sizing of analog electrical circuits, and the automatic synthesis of the topology, sizing, placement, and routing of electrical circuits (Koza and Bennett 1999).

Genetic programming has recently been used to create controllers that outperformed controllers designed using conventional approaches in the field of control using the criteria specified in the original publication (Koza, Keane, Bennett, Yu, Mydlowec, and Stiffelman 1999; Koza, Keane, Yu, Bennett, and Mydlowec 1999, 2000).

Both genetic algorithms and genetic programming have been successfully used for synthesizing controllers (Crawford, Cheng, and Menon 1999; Dewell and Menon 1999; Man, Tang, Kwong, and Halang 1997, 1999; Menon, Yousefpor, Lam, and Steinberg 1995; Sweriduk, Menon, and Steinberg 1998, 1999).

Additional information on genetic programming can be found in books such as Banzhaf, Nordin, Keller, and Francone 1998; books such as Langdon 1998, Ryan 1999, and Wong and Leung 2000 in the series on genetic programming from Kluwer Academic Publishers; in edited collections of papers such as the Advances in Genetic Programming series of books from the MIT Press (Kinnear 1994; Angeline and Kinnear 1996; Spector, Langdon, O'Reilly, and Angeline 1999); in the proceedings of the Genetic Programming Conference (Koza, Goldberg, Fogel, and Riolo 1996; Koza, Deb, Dorigo, Fogel, Garzon, Iba, and Riolo 1997; Koza, Banzhaf, Chellapilla, Deb, Dorigo, Fogel, Garzon, Goldberg, Iba, and Riolo 1998; in the proceedings of the annual Euro-GP conference (Banzhaf, Poli, Schoenauer, and Fogarty 1998; Poli, Nordin, Langdon, and Fogarty 1999); in the proceedings of the annual Genetic and Evolutionary Computation Conference (Banzhaf, Daida, Eiben, Garzon, Honavar, Jakiela, and Smith 1999); at web sites such as www.geneticprogramming.org; and in the Genetic Programming and Evolvable Machines journal (from Kluwer Academic Publishers).

\section{Genetic Programming and Control}

Controllers can be represented by block diagrams in which the blocks represent signal processing functions, in which external points represent the controller's input(s) and output(s), and in which cycles in the block diagram correspond to internal feedback inside the controller. Genetic programming can be extended to the problem of creating both the topology and parameter values for a controller by establishing a mapping between the program trees used in genetic programming and the block diagrams germane to controllers.

The number of result-producing branches in the to-beevolved controller equals the number of control variables that are to be passed from the controller to the plant. Each 
result-producing branch is a composition of the functions and terminals from a specified repertoire (below).

Program trees in the population during the initial random generation (generation 0) consist only of resultproducing branch(es). Automatically defined functions are introduced incrementally (and sparingly) into the population on subsequent generations by means of the architecture-altering operations. Each automatically defined function is a composition of the functions and terminals appropriate for control problems, references to existing automatically defined functions, and (possibly) dummy variables (formal parameters) that permit parameterization of the automatically defined function. Automatically defined functions provide a mechanism for internal feedback (recursion) within the to-be-evolved controller. Automatically defined functions also provide a mechanism for reusing useful substructures.

Each branch of each program tree in the initial random population is created in accordance with a constrained syntactic structure. Each genetic operation executed by genetic programming (crossover, mutation, reproduction, or architecture-altering operation) produces offspring that comply with the constrained syntactic structure.

\section{$5 \quad$ Preparatory Steps}

Six major preparatory steps are required before applying genetic programming: (1) determine the architecture of the program trees, (2) identify the terminals, (3) identify the functions, (4) define the fitness measure, (5) choose control parameters for the run, and (6) choose the termination criterion and method of result designation.

\subsection{Program Architecture}

Since there is one result-producing branch in the program tree for each output from the controller and this problem involves a one-output controller, each program tree has one result-producing branch. Each program tree in the initial random generation (generation 0) has no automatically defined functions. However, in subsequent generations, architecture-altering operations may insert and delete automatically defined functions (up to a maximum of five per program tree).

\section{$5.2 \quad$ Terminal Set}

A constrained syntactic structure permits only a single perturbable numerical value to appear as the argument for establishing each numerical parameter value for each signal processing block requiring a parameter value. These numerical values initially range from -5.0 to +5.0 . These numerical values are perturbed during the run by a Gaussian mutation operation that operates only on numerical values. Numerical constants are later interpreted on a logarithmic scale so that they represent values in a range of 10 orders of magnitude (Koza, Bennett, Andre, and Keane 1999).

The remaining terminals are time-domain signals. The terminal set, $\mathrm{T}$, for the result-producing branch and any automatically defined functions (except for the perturbable numerical values mentioned above) is

$T=\{$ REFERENCE_SIGNAL, CONTROLLER_OUTPUT, PLANT_OUTPUT, CONSTANT_0\}.

Space does not permit a detailed description of the the above terminals (although the function of the above terminals should be clear from their names). See Koza, Keane, Yu, Bennett, and Mydlowec 2000 for details.

5.3

\section{Function Set}

The functions are signal processing functions that operate on time-domain signals (the terminals in T). The function set, F, for the result-producing branch and any automatically defined functions is

$$
\begin{aligned}
F=\{\text { GAIN, INVERTER, LEAD, LAG, LAG2, } \\
\\
\text { DIFFERENTIAL_INPUT_INTEGRATOR, } \\
\text { DIFFERENTIATOR, ADD_SIGNAL, } \\
\\
\text { SUB_SIGNAL, ADD_3_SIGNAL, DELAY, } \\
\text { ADF0, ..,ADF } 4 .
\end{aligned}
$$

ADF $0, \ldots, A D F 4$ denote automatically defined functions added dynamically during the run by architecture-altering operations.

The names of the above functions suggest their functions; they are described in detail in Koza, Keane, Yu, Bennett, and Mydlowec 2000.

\subsection{Fitness}

Genetic programming is a probabilistic algorithm that searches the space of compositions of the available functions and terminals. The search is guided by a fitness measure. The fitness measure is a mathematical implementation of the high-level requirements of the problem. The fitness measure is couched in terms of "what needs to be done" - not "how to do it."

The fitness measure may incorporate any measurable, observable, or calculable behavior or characteristic or combination of behaviors or characteristics.

The fitness measure for most problems of controller design is multi-objective in the sense that there are several different (usually conflicting) requirements for the controller.

The fitness of each individual in the population is determined by executing the program tree (i.e., the resultproducing branch plus any automatically defined functions that may have been created during the run by the architecture-altering operations). The execution of the program tree produces an interconnected sequence of signal processing blocks - that is, a block diagram for the individual controller. The controller is embedded into a framework containing the (fixed) plant and the (fixed) external feedback loop. A SPICE netlist is then constructed to represent the block diagram of the controller, the (fixed) plant, and the (fixed) external feedback loop. This SPICE netlist is wrapped inside an appropriate set of SPICE commands to carry out various SPICE analyses in the time domain (described below). We also provide SPICE with subcircuit definitions to implement all the signal processing functions in the function set (described above) and all the signal 
processing functions necessary to represent the plant. The controller is then simulated using our modified version of the original 217,000-line SPICE3 simulator (Quarles, Newton, Pederson, and Sangiovanni-Vincentelli 1994). Our modified version of SPICE is run as a submodule within our genetic programming system. The SPICE simulator returns tabular output (representing the plant output in the time domain). An interface communicates this information to our genetic programming code.

The fitness of a controller is measured using 13 elements. These 13 elements consist of 12 time-domainbased elements based on a modified integral of timeweighted absolute error (ITAE) and one time-domainbased element measuring disturbance rejection.

The fitness of an individual controller is the sum of the (detrimental) contributions of these 13 elements of the fitness measure. The smaller the sum, the better.

The first 12 elements of the fitness measure evaluate how quickly the controller causes the plant to reach the reference signal and the controller's success in avoiding overshoot. Two reference signals are used. The first reference signal is a step function that rises from 0 to 1 volts at $t=100$ milliseconds while the second rises from 0 to 1 microvolts at $t=100$ milliseconds. The two step functions are used to deal with the non-linearity caused by the limiter. Two values of the time constant, $\tau$, are used (namely 0.5 and 1.0). Three values of $K$ are used, namely $0.9,1.0$, and 1.1 . Exposing genetic programming to different combinations of values of step size, $K$, and $\tau$ produces a robust controllers and also prevents genetic programming from engaging in pole elimination. For each of these 12 fitness cases, a transient analysis is performed in the time domain using the SPICE simulator.

Table 1 shows the elements of the fitness measure in its left-most four columns.

The contribution to fitness for each of these 12 elements of the fitness measure is based on the integral of time-weighted absolute error (ITAE)

$$
\int_{t=5}^{36}(t-5)|e(t)| A(e(t)) B C d t .
$$

Because of the built-in five-second time delay, the integration runs from time $t=5$ seconds to $t=36$ seconds. Here $e(t)$ is the difference (error) at time $t$ between the delayed plant output and the reference signal.

In implementing the above integral of time-weighted absolute error, we used a discrete approximation to the integral by considering 120300 -millisecond time steps between $t=5$ to $t=36$ seconds.

In addition, we multiplied each fitness case by the reciprocal of the amplitude of the reference signals so that both reference signals ( 1 microvolt and 1 volt) are equally influential. Specifically, $B$ is a factor that is used to normalize the contributions associated with the two step functions. $B$ multiplies the difference $e(t)$ associated with the 1-volt step function by 1 and multiplies the difference $e(t)$ associated with the 1-microvolt step function by $10^{6}$.
Also, the integral contains an additional weight, $A$, that varies with $e(t)$. The function $A$ weights all variation up to $102 \%$ of the reference signal by a factor of 1.0 , and heavily penalizes overshoots over $2 \%$ by a factor 10.0 .

Finally, the above integral contains a special weight, $C$, which is 5.0 for the two fitness cases for which $K=1$ and $\tau=1$, and 1.0 otherwise.

The 13th element of the fitness measure is based on disturbance rejection. The penalty is computed based on a time-domain analysis for 36.0 seconds. In this analysis, the reference signal is held at a value of 0 . A disturbance signal consisting of a unit step is added to the CONTROLLER_OUTPUT at time $t=0$ and the resulting disturbed signal is provided as input to the plant. The detrimental contribution to fitness is 500/36 times the time required to bring the plant output to within 20 millivolts of the reference signal of 0 volts (i.e., to reduce the effect to within $2 \%$ of the 1 -volt disturbance signal) assuming that the plant settles to within this range within 36 seconds. If the plant does not settle to within this range within 36 seconds, the detrimental contribution to fitness is 500 plus the absolute value of the plant output in volts times 500. For example, if the effect of the disturbance was never reduced below 1 volts, the detrimental contribution to fitness would be 1000 .

A controller that cannot be simulated by SPICE is assigned a high penalty value of fitness $\left(10^{8}\right)$.

\subsection{Control Parameters}

The population size, $M$, was 500,000. A maximum size of 150 points (functions and terminals) was established for each result-producing branch and a maximum size of 100 points was established for each automatically defined function. The other parameters for controlling the runs are the default values that we apply to a broad range of problems (Koza, Bennett, Andre, and Keane 1999).

\subsection{Termination}

The run was manually monitored and manually terminated when the fitness of many successive best-ofgeneration individuals appeared to have reached a plateau. The single best-so-far individual is harvested and designated as the result of the run.

\subsection{Parallel Implementation}

This problem was run on a home-built Beowulf-style (Sterling, Salmon, Becker, and Savarese 1999; Bennett, Koza, Shipman, and Stiffelman 1999) parallel cluster computer system consisting of 1,000 $350 \mathrm{MHz}$ Pentium II processors (each accompanied by 64 megabytes of RAM). The system has a $350 \mathrm{MHz}$ Pentium II computer as host. The processing nodes are connected with a 100 megabit-per-second Ethernet. The processing nodes and the host use the Linux operating system. The distributed genetic algorithm with unsynchronized generations and semi-isolated subpopulations was used with a subpopulation size of $Q=500$ at each of $D=1,000$ demes. Two processors are housed in each of the 500 physical boxes of the system. As each processor (asynchronously) completes a generation, four boatloads 
of emigrants from each subpopulation (selected probabilistically based on fitness) are dispatched to each of the four toroidally adjacent processors. The migration rate is $2 \%$ (but $10 \%$ if the toroidally adjacent node is in the same physical box). 


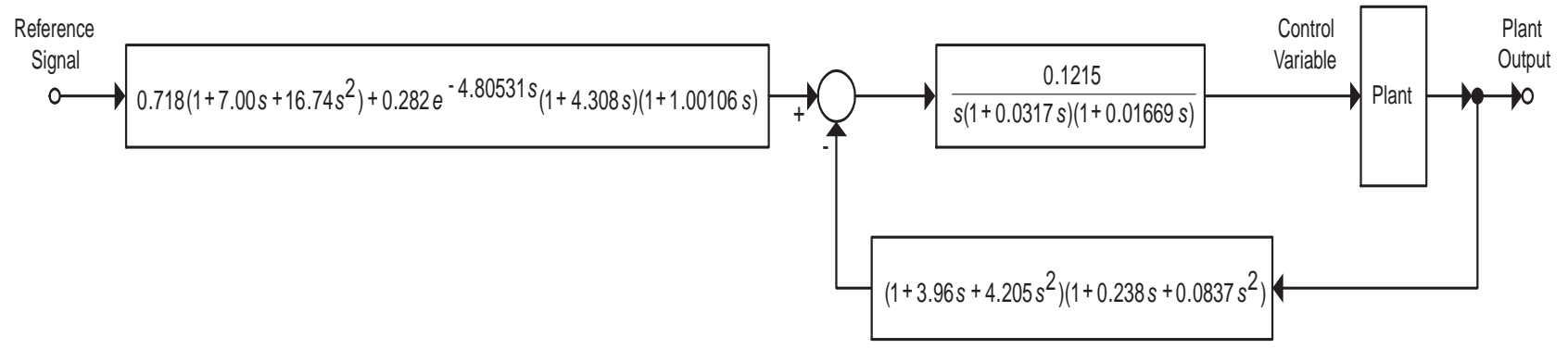

Figure 1 Best-of-run controller from generation 129 for three-lag plant with five-second delay.

\section{$6 \quad$ Results}

The best individual in generation 0 has a fitness of 1926.498 .

The best-of-run controller emerged in generation 129 (figure 1). This best-of-run controller has a fitness of 522.605. The result-producing branch of this best-of-run individual has 119 points (functions and terminals) and 95, 93, and 70 points, respectively, in its three automatically defined functions. Note that genetic programming employed a 4.8 second delay (comparable to the five-second plant delay) in the transfer function of the evolved pre-filter. This best-of-run controller from generation 129 has a better value of fitness for a step size of 1 volt, an internal gain, $K$, of 1.0 , and a time-constant, $\tau$,of 1.0 (the specific case considered by Astrom and Hagglund 1995).

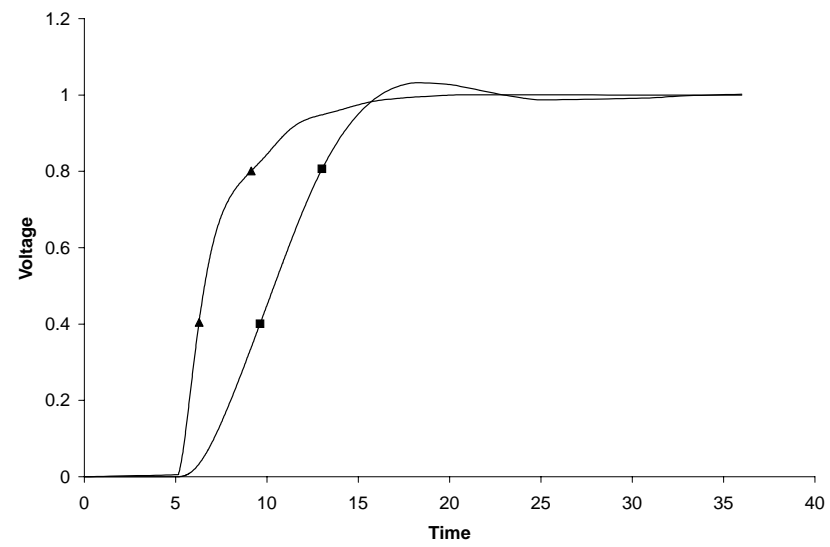

Figure 2 Comparison for step input.
Figure 2 compares the time-domain response to step input of the best-of-run controller from generation 129 (triangles) with the controller in Astrom and Hagglund 1995 (squares) for a step size of 1 volt, an internal gain, $K$, of 1.0, and a time-constant, $\tau$,of 1.0.

Figure 3 compares the disturbance rejection of the best-of-run controller from generation 129 (triangles) with the controller in Astrom and Hagglund 1995 (squares) for a step size of 1 volt, an internal gain, $K$, of 1.0 , and a time-constant, $\tau$,of 1.0 .

Table 1 compares the fitness of the best-of-run controller from generation 129 and the Astrom and Hagglund 1995. Two of the entries are divided by the special weight $C=5.0$. All 13 entries are better for the genetically evolved controller than for the Astrom and Hagglund 1995 controller.

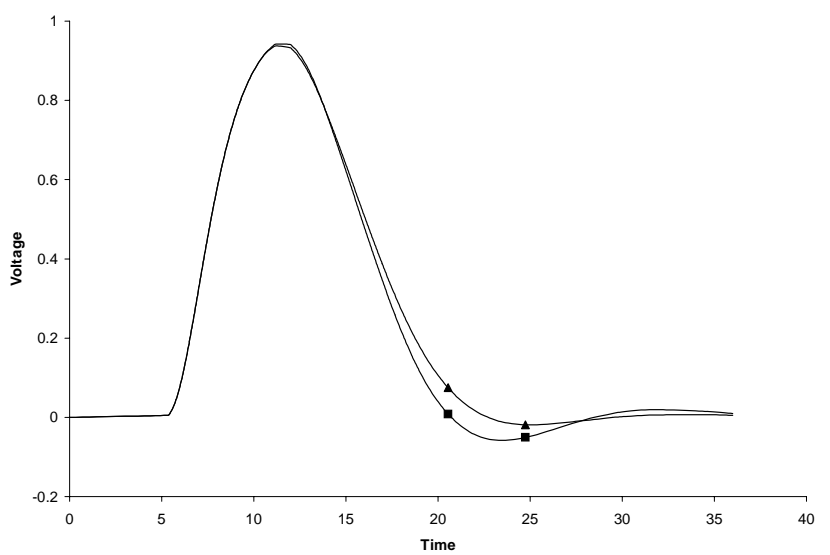

Figure 3 Comparison for disturbance rejection. 
Table 1 Fitness of two controllers for three-lag plant with five-second delay

\begin{tabular}{|l|l|l|l|l|l|}
\hline Element & $\begin{array}{l}\text { Step } \\
\text { size } \\
\text { (volts) }\end{array}$ & $\begin{array}{l}\text { Plant } \\
\text { internal } \\
\text { Gain, } K\end{array}$ & $\begin{array}{l}\text { Time } \\
\text { constan } \\
\text { t, } \tau\end{array}$ & $\begin{array}{l}\text { Fitness of } \\
\text { best-of-run } \\
\text { generation } \\
129\end{array}$ & $\begin{array}{l}\text { Fitness of } \\
\text { Astrom and } \\
\text { Hagglund } \\
\text { controller }\end{array}$ \\
\hline 0 & 1 & 0.9 & 1.0 & 13.7 & 27.4 \\
\hline 1 & 1 & 0.9 & 0.5 & 25.6 & 38.2 \\
\hline 2 & 1 & 1.0 & 1.0 & $34.0 / 5=6.8$ & 22.9 \\
\hline 3 & 1 & 1.0 & 0.5 & 18.6 & 29.3 \\
\hline 4 & 1 & 1.1 & 1.0 & 4.4 & 25.4 \\
\hline 5 & 1 & 1.1 & 0.5 & 16.3 & 22.7 \\
\hline 6 & $10^{-6}$ & 0.9 & 1.0 & 13.2 & 27.4 \\
\hline 7 & $10^{-6}$ & 0.9 & 0.5 & 25.5 & 38.2 \\
\hline 8 & $10^{-6}$ & 1.0 & 1.0 & $30.7 / 5=6.1$ & 22.9 \\
\hline 9 & $10^{-6}$ & 1.0 & 0.5 & 18.5 & 29.3 \\
\hline 10 & $10^{-6}$ & 1.1 & 1.0 & 4.3 & 25.4 \\
\hline 11 & $10^{-6}$ & 1.1 & 0.5 & 16.2 & 22.7 \\
\hline Disturbance & 1 & 1 & 1 & 302 & 373 \\
\hline
\end{tabular}

\section{References}

Angeline, Peter J. and Kinnear, Kenneth E. Jr. (editors). 1996. Advances in Genetic Programming 2. Cambridge, MA: The MIT Press.

Astrom, Karl J. and Hagglund, Tore. 1995. PID Controllers: Theory, Design, and Tuning. 2nd Edition. Research Triangle Park, NC: Instrument Society of America.

Banzhaf, Wolfgang, Daida, Jason, Eiben, A. E., Garzon, Max H., Honavar, Vasant, Jakiela, Mark, and Smith, Robert E. (editors). 1999. GECCO-99: Proceedings of the Genetic and Evolutionary Computation Conference, July 13-17, 1999, Orlando, Florida USA. San Francisco, CA: Morgan Kaufmann.

Banzhaf, Wolfgang, Nordin, Peter, Keller, Robert E., and Francone, Frank D. 1998. Genetic Programming - An Introduction. San Francisco, CA: Morgan Kaufmann and Heidelberg: dpunkt.

Banzhaf, Wolfgang, Poli, Riccardo, Schoenauer, Marc, and Fogarty, Terence C. 1998. Genetic Programming: First European Workshop. EuroGP'98. Paris, France, April 1998 Proceedings. Paris, France. April 1998. Lecture Notes in Computer Science. Volume 1391. Berlin, Germany: Springer-Verlag.

Bennett, Forrest H III, Koza, John R., Shipman, James, and Stiffelman, Oscar. 1999. Building a parallel computer system for $\$ 18,000$ that performs a half petaflop per day. In Banzhaf, Wolfgang, Daida, Jason, Eiben, A. E., Garzon, Max H., Honavar, Vasant, Jakiela, Mark, and Smith, Robert E. (editors). 1999. GECCO-99: Proceedings of the Genetic and Evolutionary Computation Conference, July 13-17, 1999, Orlando, Florida USA. San Francisco, CA: Morgan Kaufmann. 1484 - 1490.
Boyd, S. P. and Barratt, C. H. 1991. Linear Controller Design: Limits of Performance. Englewood Cliffs, NJ: Prentice Hall.

Crawford, L. S., Cheng, V. H. L., and Menon, P. K. 1999. Synthesis of flight vehicle guidance and control laws using genetic search methods. Proceedings of 1999 Conference on Guidance, Navigation, and Control. Reston, VA: American Institute of Aeronautics and Astronautics. Paper AIAA-99-4153.

Dewell, Larry D. and Menon, P. K. 1999. Low-thrust orbit transfer optimization using genetic search. Proceedings of 1999 Conference on Guidance, Navigation, and Control. Reston, VA: American Institute of Aeronautics and Astronautics. Paper AIAA-99-4151.

Holland, John H. 1975. Adaptation in Natural and Artificial Systems. Ann Arbor, MI: University of Michigan Press.

Kinnear, Kenneth E. Jr. (editor). 1994. Advances in Genetic Programming. Cambridge, MA: The MIT Press.

Koza, John R. 1992. Genetic Programming: On the Programming of Computers by Means of Natural Selection. Cambridge, MA: MIT Press.

Koza, John R. 1994a. Genetic Programming II: Automatic Discovery of Reusable Programs. Cambridge, MA: MIT Press.

Koza, John R. 1994b. Genetic Programming II Videotape: The Next Generation. Cambridge, MA: MIT Press.

Koza, John R., Banzhaf, Wolfgang, Chellapilla, Kumar, Deb, Kalyanmoy, Dorigo, Marco, Fogel, David B., Garzon, Max H., Goldberg, David E., Iba, Hitoshi, and Riolo, Rick. (editors). 1998. Genetic Programming 
1998: Proceedings of the Third Annual Conference. San Francisco, CA: Morgan Kaufmann.

Koza, John R., and Bennett III, Forrest H. 1999. Automatic synthesis, placement, and routing of electrical circuits by means of genetic programming. In Spector, Lee, Langdon, William B., O'Reilly, UnaMay, and Angeline, Peter (editors). Advances in Genetic Programming 3. Cambridge, MA: MIT Press. Chapter 6. Pages 105 - 134.

Koza, John R., Bennett III, Forrest H, Andre, David, and Keane, Martin A. 1999. Genetic Programming III: Darwinian Invention and Problem Solving. San Francisco, CA: Morgan Kaufmann. Forthcoming.

Koza, John R., Bennett III, Forrest H, Andre, David, Keane, Martin A., and Brave Scott. 1999. Genetic Programming III Videotape: Human-Competitive Machine Intelligence. San Francisco, CA: Morgan Kaufmann.

Koza, John R., Deb, Kalyanmoy, Dorigo, Marco, Fogel, David B., Garzon, Max, Iba, Hitoshi, and Riolo, R. L. (editors). 1997. Genetic Programming 1997: Proceedings of the Second Annual Conference San Francisco, CA: Morgan Kaufmann.

Koza, John R., Goldberg, David E., Fogel, David B., and Riolo, Rick L. (editors). 1996. Genetic Programming 1996: Proceedings of the First Annual Conference. Cambridge, MA: MIT Press.

Koza, John R., Keane, Martin A., Bennett, Forrest H III, $\mathrm{Yu}$, Jessen, Mydlowec, William, and Stiffelman, Oscar. 1999. Automatic creation of both the topology and parameters for a robust controller by means of genetic programming. Proceedings of the 1999 IEEE International Symposium on Intelligent Control, Intelligent Systems, and Semiotics. Piscataway, NJ: IEEE. Pages 344 - 352.

Koza, John R., Keane, Martin A., Yu, Jessen, Bennett, Forrest H III, Mydlowec, William, and Stiffelman, Oscar. 1999. Automatic synthesis of both the topology and parameters for a robust controller for a nonminimal phase plant and a three-lag plant by means of genetic programming. Proceedings of 1999 IEEE Conference on Decision and Control. Pages 5292 5300.

Koza, John R., Keane, Martin A., Yu, Jessen, Bennett, Forrest H III, and Mydlowec, William. 2000. Automatic creation of human-competitive programs and controllers by means of genetic programming. Genetic Programming and Evolvable Machines. (1) $121-164$.

Koza, John R., and Rice, James P. 1992. Genetic Programming: The Movie. Cambridge, MA: MIT Press.

Langdon, William B. 1998. Genetic Programming and Data Structures: Genetic Programming + Data Structures = Automatic Programming! Amsterdam: Kluwer.

Man, K. F., Tang, K. S., Kwong, S., and Halang, W. A. 1997. Genetic Algorithms for Control and Signal Processing. London: Springer-Verlag.
Man, K. F., Tang, K. S., Kwong, S., and Halang, W. A. 1999. Genetic Algorithms: Concepts and Designs. London: Springer-Verlag.

Menon, P. K., Yousefpor, M., Lam, T., and Steinberg, M. L. 1995. Nonlinear flight control system synthesis using genetic programming. Proceedings of 1995 Conference on Guidance, Navigation, and Control. Reston, VA: American Institute of Aeronautics and Astronautics. Pages 461 - 470.

Poli, Riccardo, Nordin, Peter, Langdon, William B., and Fogarty, Terence C. 1999. Genetic Programming: Second European Workshop. EuroGP'99. Proceedings. Lecture Notes in Computer Science. Volume 1598. Berlin: Springer-Verlag.

Quarles, Thomas, Newton, A. R., Pederson, D. O., and Sangiovanni-Vincentelli, A. 1994. SPICE 3 Version $3 F 5$ User's Manual. Department of Electrical Engineering and Computer Science, Univ. of California. Berkeley, CA. March 1994.

Ryan, Conor. 1999. Automatic Re-engineering of Software Using Genetic Programming. Amsterdam: Kluwer Academic Publishers.

Spector, Lee, Langdon, William B., O'Reilly, Una-May, and Angeline, Peter (editors). 1999. Advances in Genetic Programming 3. Cambridge, MA: MIT Press.

Sterling, Thomas L., Salmon, John, Becker, D. J., and Savarese, D. F. 1999. How to Build a Beowulf: A Guide to Implementation and Application of $P C$ Clusters. Cambridge, MA: MIT Press.

Sweriduk, G. D., Menon, P. K., and Steinberg, M. L. 1998. Robust command augmentation system design using genetic search methods. Proceedings of 1998 Conference on Guidance, Navigation, and Control. Reston, VA: American Institute of Aeronautics and Astronautics. Pages $286-294$.

Sweriduk, G. D., Menon, P. K., and Steinberg, M. L. 1999. Design of a pilot-activated recovery system using genetic search methods. Proceedings of 1998 Conference on Guidance, Navigation, and Control. Reston, VA: American Institute of Aeronautics and Astronautics.

Wong, Man Leung and Leung, Kwong Sak. 2000. Data Mining Using Grammar Based Genetic Programming and Applications. Amsterdam: Kluwer Academic Publishers. 